\title{
Ranking income distributions: a rank-dependent and needs-based approach
}

\author{
Ida Petrillo
}

SERIES Working PAPERS N. 03/2017

SERIES sono pubblicati a cura del Dipartimento di Economia e Finanza dell'Università degli Studi di Bari "Aldo Moro". I lavori riflettono esclusivamente le opinioni degli autori e non impegnano la responsabilità del Dipartimento. SERIES vogliono promuovere la circolazione di studi ancora preliminari e incompleti, per suscitare commenti critici e suggerimenti. Si richiede di tener conto della natura provvisoria dei lavori per eventuali citazioni o per ogni altro uso.
SERIES are published under the auspices of the Department of Economics and Finance of the University of Bari. Any opinions expressed here are those of the authors and not those of the Department. Often SERIES divulge preliminary or incomplete work, circulated to favor discussion and comment. Citation and use of these paper should consider their provisional character. 


\title{
Ranking income distributions: a rank-dependent and needs-based approach*
}

\author{
Ida Petrillo ${ }^{\dagger}$
}

July 18, 2017

\begin{abstract}
This paper provides a normative framework to assess social welfare within a rankdependent and bidimensional setting where individuals are characterized by income and needs. In line with the growing interest in top incomes and inequalities concerning the upper part of the income distribution (see Atkinson and Piketty, 2007; 2010; Aaberge, Havnes and Mogstad, 2013), we propose a complete framework to rank bidimensional distributions, by focussing on inequalities among the poorest as well as the richest individuals, both in the monetary and non-monetary dimension. To this end, we develop third degree inverse stochastic dominance conditions for classes of social welfare functions satisfying: i) downside inequality aversion with respect to income and upside inequality aversion with respect to needs; ii) upside inequality aversion with respect to income and downside inequality aversion with respect to needs; iii) upside inequality aversion both with respect to income and needs. Our results emerge along with the existing one, formulated by Zoli (2000), supporting downside inequality aversion both with respect to income and needs.
\end{abstract}

Jel classification: D31, D63, I31

Keywords: sequential stochastic dominance; social welfare; inequality

${ }^{*}$ I am very grateful to my PhD supervisor, Vito Peragine, for invaluable advice and fruitful discussions. I would also like to thank Flaviana Palmisano for precious suggestions, Giovanni Taglialatela for helpful comments and the audience of the seminar held in the Department of Economics and Mathematical Methods, University of Bari. This paper will be presented at the XXIX annual conference of the Italian Society of Public Economics (SIEP), 21-22 September 2017, Catania (Italy).

${ }^{\dagger}$ University of Bari - Department of Economics and Finance. E-mail: ida.petrillo@uniba.it 


\title{
Ranking income distributions: a rank-dependent and needs-based approach*
}

\author{
Ida Petrillo ${ }^{\dagger}$
}

July 18, 2017

\begin{abstract}
This paper provides a normative framework to assess social welfare within a rankdependent and bidimensional setting where individuals are characterized by income and needs. In line with the growing interest in top incomes and inequalities concerning the upper part of the income distribution (see Atkinson and Piketty, 2007; 2010; Aaberge, Havnes and Mogstad, 2013), we propose a complete framework to rank bidimensional distributions, by focussing on inequalities among the poorest as well as the richest individuals, both in the monetary and non-monetary dimension. To this end, we develop third degree inverse stochastic dominance conditions for classes of social welfare functions satisfying: i) downside inequality aversion with respect to income and upside inequality aversion with respect to needs; ii) upside inequality aversion with respect to income and downside inequality aversion with respect to needs; iii) upside inequality aversion both with respect to income and needs. Our results emerge along with the existing one, formulated by Zoli (2000), supporting downside inequality aversion both with respect to income and needs.
\end{abstract}

Jel classification: D31, D63, I31

Keywords: sequential stochastic dominance; social welfare; inequality

${ }^{*}$ I am very grateful to my PhD supervisor, Vito Peragine, for invaluable advice and fruitful discussions. I would also like to thank Flaviana Palmisano for precious suggestions, Giovanni Taglialatela for helpful comments and the audience of the seminar held in the Department of Economics and Mathematical Methods, University of Bari. This paper will be presented at the XXIX annual conference of the Italian Society of Public Economics (SIEP), 21-22 September 2017, Catania (Italy).

${ }^{\dagger}$ University of Bari - Department of Economics and Finance. E-mail: ida.petrillo@uniba.it 


\section{Introduction}

Since the pioneering contribution of Atkinson and Bourguignon (1987), sequential stochastic dominance techniques have become a widely accepted criterion for ranking income distributions of populations decomposed into subgroups of individuals homogeneous in needs. Such techniques take into account the multidimensional nature of well-being and social welfare and allow to rank different distributions by including both the monetary and some non-monetary attribute characterizing the populations under scrutiny. In particular, the authors depart from the unanimous preference expressed by an additively separable social welfare function of the utilitarian type. By imposing certain conditions on the derivatives of the utility function, they obtain different classes of SWFs encompassing some socially desirable properties. For each class of SWFs introduced, it is possible to assess welfare dominance by means of readily implementable tests, namely first and second order sequential stochastic dominance conditions. The usefulness of this framework paves the way to a fertile strand of research, whose applications may be distinguished considering whether they adopt the utilitarian or rank-dependent representation of social preferences. In particular, as far as the utilitarian framework is concerned, Jenkins and Lambert (1993), differently from Atkinson and Bourguignon (1987), allow for demographic differences; Chambaz and Maurin (1998) introduce additional sequential procedures; Lambert and Ramos (2002) develop third order sequential stochastic dominance conditions for social welfare functions satisfying Kolm's principle of diminishing transfers. Conversely, in the rank-dependent framework, Zoli (2000) develops inverse sequential dominance conditions of first, second and third order, for classes of social welfare functions satisfying, in the last case, a positional version of the transfer sensitivity principle. The last two mentioned works, dealing with third order sequential dominance, despite being based respectively on an utilitarian and rank-dependent ground, share the common feature of being concerned about differences occurring in the lower part of the distribution, thus endorsing what has been called in Aaberge, Havnes and Mogstad (2013) downside inequality aversion.

However, recent developments in the inequality literature are raising a growing interest in top incomes and the evolution of inequalities concerning the upper part of the income distribution (see Atkinson and Piketty, 2007; 2010), leading the researchers to reconsider their standard attitude towards downside inequality aversion, which is encompassed by a social welfare function satisfying the principle of diminishing trasfer within the utilitarian approach, and the principle of downside positional transfer sensitivity within the 
rank-dependent setting. In this vein, Aaberge (2009) introduces the principle of upside positional transfer sensitivity and Aaberge et al. (2013) develop a theory for unambiguously ranking income distributions. In their paper, referred to a unidimensional context based on income only, they develop a third order inverse downward dominance condition, that places more emphasis on differences in the upper part of the distribution, and holds for classes of social welfare functions satisfying the principle of upside positional transfer sensitivity $^{1}$. This condition endorses exactly the opposite view with respect to the standard dominance condition of third degree, renamed upward dominace, that places more emphasis on differences in the lower part of the distribution and holds for classes of social welfare functions satisfying the principle of downside positional transfer sensitivity.

In the present work, we draw togeher the arguments introduced above, namely the concern for the multidimensional evaluation of social welfare and the interest in inequalities affecting the top of the distribution. To this end, we develop third-degree inverse stochastic dominance conditions suitable for a rank-dependent and bidimensional framework and able to encompass different attitudes towards inequality aversion. In particular, a greater attention may be devoted to inequalities arising among the poorest as well as the richest individuals of the population, both in a monetary and a non-monetary sense. To be more precise, we develop third degree inverse dominance conditions for classes of social welfare functions satisfying:

i) downside inequality aversion with respect to income and upside inequality aversion with respect to needs;

ii) upside inequality aversion with respect to income and downside inequality aversion with respect to needs;

iii) upside inequality aversion both with respect to income and needs.

These results emerge along with the existing one, formulated by Zoli (2000), supporting downside inequality aversion both with respect to income and needs.

Notice that in all these cases the standard Pigou-Dalton transfer principle is preserved, what differs from one approach to the other is whether one puts more emphasis on differences among the richest or the poorest individuals, having in mind that, within our setting, each individual is characterized by a monetary and a non-monetary attribute.

A remark is in order at this point. The standard practise adopted in sequential dominance tests consists in dividing the population into subgroups depending on family size,

\footnotetext{
${ }^{1}$ See Aaberge and Peluso (2012) on second-degree upward and downward dominance to rank multidimensional distributions according to their extent of deprivation.
} 
age, type of housing. However, as noted by Duclos and Échevin (2009), the method may also cover cases where this information is replaced by any other ordinal variable. Hence, also the framework we analyze can be naturally extended in order to consider individuals differing in some non-income dimension such as health, education and so on. However, throughout the paper, we use the generic term "needs", having in mind that it has a quite flexible and wide content.

This contribution represents an additional instrument in the researcher's toolbox to help evaluating different distributions of income and needs, in particular when there is interest in embracing not only the traditional view of giving higher weight to inequalities at the bottom of the distribution, but also the more recent one of looking at the top of it.

The remainder of the paper is organized as follows: Section 2 outlines the framework, Section 3 presents the theoretical results, Section 4 concludes.

\section{Theoretical model}

\subsection{Notation}

Let individuals $s$, belonging to population $S$, be described by an income level $y$ and by an additional non-monetary dimension of well-being, that we indicate with the generic term "needs". Consider the population $S$ as partitioned into groups $S_{i}, i=1, \ldots, n$, characterized by a decreasing level of these needs, in such a way that we can consider group $i$ to be needier and consequently poorer in a non-monetary sense than group $i+1$. We assume that the needs' level of individuals gives an extra information about their identity to be read together with the traditional information about monetary incomes. If $F_{i}(y)$ is the cumulative income distribution of group $i$ and we denote by $q_{i}^{F}$ the share of individuals belonging to group $i$, we have that the overall cumulative income distribution is $F(y)=\sum_{i=1}^{n} q_{i}^{F} F_{i}(y)$. Let $\mu(F)$ be the mean income of distribution $F$. Moreover, let $\digamma$ be the set of all such cumulative distributions and $F_{i}^{-1}(p)=\inf \left\{y: F_{i}(y) \geq p\right\}$ with $p \in[0,1]$ the left continuous inverse of $F_{i}(y)$ denoting the income $y$ of an individual at the $p^{\text {th }}$ percentile of the distribution of group $i$.

We are interested in a formulation of the rank-dependent social welfare function that is able to capture not only the extent of monetary wealth, but also its non-monetary side, proxied by the membership of individuals to different needs-based groups. According to 
this idea, a rank-dependent needs-based SWF could be expressed by:

$$
W(F)=\sum_{i=1}^{n} q_{i}^{F} \int_{0}^{1} v_{i}(p) F_{i}^{-1}(p) d p
$$

where $v_{i}(p)$ is the weight attached to the income of an individual ranked at the $p^{\text {th }}$ percentile in group $i$, which we assume to be a continuous and twice differentiable function.

It is clear that the aggregation of incomes encompasses two different weighting procedures: on the one hand, within each group, a weighted average of the incomes of individuals is obtained on the basis of the their position in the income ranking; on the other hand, incomes of each group, weighted by the relevant population share, are aggregated according to weights that are specific for each group.

Restrictions on weights will define different classes of social welfare functions, characterized by different normative implications.

\section{$2.2 \quad$ Properties}

In this section, we discuss different restrictions on the weight function. By imposing a greater number of such restrictions, although we make stricter assumptions about the properties of the social welfare function, we will be able to perform more comparisons between intersecting distribution functions.

Each property on the set of weights will be explained and justified by means of various refinements of the Pigou-Dalton transfer principle.

Property 1. (Welfare monotonicity)

$$
v_{i}(p) \geq 0 \text { for any } i=1, \ldots, n, \text { for any } p \in[0,1]
$$

First of all, we impose a standard monotonicity assumption, requiring that social welfare is a nondecreasing function of individuals' incomes. This amounts to specifying nonnegative weights.

Property 2. (Concern for needs)

$$
v_{i}(p) \geq v_{i+1}(p) \text { for any } i=1, \ldots, n-1, \text { for any } p \in[0,1]
$$

We are interested in a welfare representation that gives priority to individuals exhibiting 
higher needs, that is, if two individuals belonging to different needs groups are ranked at the same percentile in the income distribution of their respective group, the social planner should give higher weight to the income of the needier individual.

A class of social welfare functions satisfying both Properties 1 and 2 displays weights such that:

$$
v_{i}(p) \geq v_{i+1}(p) \geq 0 \text { for any } i=1, \ldots, n-1, \text { for any } p \in[0,1]
$$

Property 3. (Inequality aversion within groups)

$$
v_{i}^{\prime}(p) \leq 0 \text { for any } i=1, \ldots, n, \text { for any } p \in[0,1]
$$

This property corresponds to the standard principle of transfers applied over homogeneous populations. In particular, we restrict our attention to individuals belonging to the same group, i.e. characterized by the same level of needs. The social planner should display inequality aversion, thus evaluating more a distribution where the incomes of individuals of the same group are more equal. In fact, imposing negative first derivatives on the weight function $v_{i}(p)$ is equivalent to impose that a progressive income transfer from indviduals belonging to group $i$ and ranked at the $(p+\pi)^{t h}$ percentile of the income distribution to individuals of the same group $i$ but ranked at the $p^{\text {th }}$ income percentile, with $\pi \geq 0$, is welfare enhancing (see Mehran, 1976 and Yaari, 1987, 1988).

Property 4. (Inequality aversion between groups)

$$
v_{i}^{\prime}(p) \leq v_{i+1}^{\prime}(p) \text { for any } i=1, \ldots, n-1, \text { for any } p \in[0,1]
$$

This property states that the same progressive transfer of income is evaluated differently if it takes place in different needs groups. Namely, $W$ increases more, the higher is the needs level of the group, within which the progressive transfer takes place. Following Zoli (2000), we consider two identical progressive transfers $\delta>0$ from individuals ranked at the $(p+\pi)^{t h}$ percentile of the income distribution to individuals belonging to the same group, but ranked at the $p^{\text {th }}$ income percentile, with $\pi \geq 0$. These transfers take place within two different groups: the former is within group $i$, while the latter is within group $i+1$, that is less needy. $W$ obeys the principle of inequality aversion between groups if the impact on social welfare of the transfer within group $i$ is greater than the impact of the same transfer applied to group $i+1$, that is: 


$$
\Delta_{i} W(p, \pi, \delta) \geq \Delta_{i+1} W(p, \pi, \delta) \quad \text { for any } p, \pi, \delta, i
$$

which corresponds to:

$$
-\delta v_{i}(p+\pi)+\delta v_{i}(p) \geq-\delta v_{i+1}(p+\pi)+\delta v_{i+1}(p) \quad \text { for any } p, \pi, \delta, i
$$

Simplifying for $\delta$, it becomes:

$$
-v_{i}(p+\pi)+v_{i}(p) \geq-v_{i+1}(p+\pi)+v_{i+1}(p) \quad \text { for any } p, \pi, i
$$

that is equivalent to:

$$
v_{i}(p+\pi)-v_{i}(p) \leq v_{i+1}(p+\pi)-v_{i+1}(p) \quad \text { for any } p, \pi, i
$$

which for small $\pi$ becomes:

$$
v_{i}^{\prime}(p) \leq v_{i+1}^{\prime}(p) \quad \text { for any } p, i
$$

Properties 3 and 4, taken together, require that:

$$
v_{i}^{\prime}(p) \leq v_{i+1}^{\prime}(p) \leq 0 \text { for any } i=1, \ldots, n-1, \text { for any } p \in[0,1]
$$

Property 5. (Within-group Downside Positional Transfer Sensitivity)

$$
v_{i}^{\prime \prime}(p) \geq 0 \text { for any } i=1, \ldots, n, \text { for any } p \in[0,1]
$$

We confine our attention to intra-group comparisons and make use of the results, due to Zoli (1999), referred to homogeneous populations. The principle we are going to use, defined in Zoli (1999) as positional transfer sensitivity, has been redifined by Aaberge, Havnes and Mogstad (2013) as downside positional transfer sensitivity (DPTS), in order to distinguish such principle from the different upside version of it.

To better understand the meaning of the DPTS, consider a fixed progressive transfer $\delta$ taking place between individuals belonging to group $i$ and showing equal difference in ranks, but located in different positions within their group's distribution. We require the transfer taking place at lower ranks to be more equalizing, and thus more welfare improving, than the transfer taking place at higher ranks. In particular, we consider two 
progressive transfers - within group $i$ - of the same amount, $\delta$, one from individuals at rank $q+\pi$ to individuals at $\operatorname{rank} q$, and another from $\operatorname{rank} p+\pi$ to $\operatorname{rank} p$, with $q \leq p$ and $\pi$ expressing the equal difference in ranks, and denote the change in social welfare associated with them, rispectively, by $\Delta_{i} W(q, \pi, \delta)$ and $\Delta_{i} W(p, \pi, \delta)$. $W$ satisfies the DPTS if and only if $\Delta_{i} W(q, \pi, \delta) \geq \Delta_{i} W(p, \pi, \delta)$ for all $q \leq p$, where $p=q+\varepsilon$. In formal terms, this means that:

$$
-\delta v_{i}(q+\pi)+\delta v_{i}(q) \geq-\delta v_{i}(p+\pi)+\delta v_{i}(p) \quad \text { for any } p, \pi, \delta, i
$$

which is equivalent to:

$$
-\delta v_{i}(q+\pi)+\delta v_{i}(q)+\delta v_{i}(p+\pi)-\delta v_{i}(p) \geq 0 \quad \text { for any } p, \pi, \delta, i
$$

Hence, we may interpret this condition as the requirement that a combination of a progressive and a regressive transfer of the same amount $\delta$ but taking place respectively from the $(q+\pi)^{t h}$ to the $q^{t h}$ percentile and from the $p^{\text {th }}$ to the $(p+\pi)^{t h}$ percentile of the income distribution, with $q \leq p$, does not lead to a welfare loss. Such combination has been called in Zoli (1999) an "Elementary Favorable Composite Positional Transfer" (EFCPT). In this context, it will be useful to refer to is as an "Elementary Downside Favorable Composite Positional Transfer" (EDFCPT), in order to distinguish it form its Upside counterpart.

We now proceed by refining the above expression in order to obtain a condition in terms of the weight function.

In particular, simplifying for $\delta$, we get that:

$$
\left[v_{i}(p+\pi)-v_{i}(p)\right]-\left[v_{i}(q+\pi)-v_{i}(q)\right] \geq 0 \quad \text { for any } p, \pi, i
$$

which, for $\pi$ small enough, becomes:

$$
v_{i}^{\prime}(p)-v_{i}^{\prime}(q) \geq 0 \quad \text { for any } p, i
$$

Having in mind that $p=q+\varepsilon$, this could be written as:

$$
v_{i}^{\prime}(q+\varepsilon)-v_{i}^{\prime}(q) \geq 0 \quad \text { for any } p, i
$$

that for small $\varepsilon$ is equivalent to: 


$$
v_{i}^{\prime \prime}(q) \geq 0 \quad \text { for any } p, i
$$

Therefore, in terms of the weight function, the principle of within-group downside positional transfer sensitivity is equivalent to impose positive second derivatives, namely: $v_{i}^{\prime \prime}(p) \geq 0$ for any $i=1, \ldots, n$, for any $p \in[0,1]$. Following Aaberge, Havnes, Mogstad (2013), an inequality averse social planner who supports the principle of DPTS is said to exhibit downside (positional) inequality aversion. Since every time we adopt the intragroup perspective, within our needs-based framework, individuals differ only in the extent of the income component, this means that our social planner could be said to exhibit downside inequality aversion with respect to income.

Property 6. (Within-group Upside Positional Transfer Sensitivity)

$$
v_{i}^{\prime \prime}(p) \leq 0 \text { for any } i=1, \ldots, n, \text { for any } p \in[0,1]
$$

We confine again our attention to intra-group comparisons and exploit the results, due to Aaberge, Havnes and Mogstad (2013), referred to homogeneous populations. The principle of upside positional transfer sensitivity (UPTS) states that the same progressive transfer from a richer to a poorer individual, with a fixed number of peolple between the donor and the receiver, is valued more if it occurs at higher income levels. Clearly, an inequality averse social planner who supports the principle of UPTS can be said to exhibit upside (positional) inequality aversion with respect to income.

To better understand the meaning of the UPTS, consider a fixed progressive transfer $\delta$ taking place between individuals with equal difference in ranks. We require the transfer taking place at lower ranks to be less equalizing, and thus less welfare improving, than the transfer taking place at higher ranks. The normative ground of this principle has to be found in the growing interest, arising among researchers, in the inequalities characterizing the incomes placed at the top of the distribution (see e.g. Atkinson and Piketty, 2007, 2010).

We consider two progressive transfers - within group $i$ - of the same amount, $\delta$, one from an individual at $\operatorname{rank} q+\pi$ to an individual at rank $q$, and another from rank $p+\pi$ to rank $p$, with $p \geq q$ and $\pi$ expressing the equal difference in ranks, and denote the change in social welfare associated with them, rispectively, by $\Delta_{i} W(q, \pi, \delta)$ and $\Delta_{i} W(p, \pi, \delta)$. W satisfies the UPTS if and only if $\Delta_{i} W(q, \pi, \delta) \leq \Delta_{i} W(p, \pi, \delta)$ for all $q \leq p$, with $p=q+\varepsilon$. In formal terms, this means that: 


$$
-\delta v_{i}(q+\pi)+\delta v_{i}(q) \leq-\delta v_{i}(p+\pi)+\delta v_{i}(p) \quad \text { for any } p, \pi, \delta, i
$$

which is equivalent to:

$$
-\delta v_{i}(p+\pi)+\delta v_{i}(p)+\delta v_{i}(q+\pi)-\delta v_{i}(q) \geq 0 \quad \text { for any } p, \pi, \delta, i
$$

Hence, we may interpret this condition as the requirement that a combination of a progressive and a regressive transfer of the same amount $\delta$, but taking place respectively from the $(p+\pi)^{t h}$ to the $p^{t h}$ percentile and from the $q^{t h}$ to the $(q+\pi)^{t h}$ percentile of the income distribution, with $p \geq q$, does not lead to a welfare loss. In the same fashion as Zoli (2000), we might call such combination an "Elementary Upside Favorable Composite Positional Transfer" (EUFCPT).

We now proceed by simplifyng the above expression in order to obtain a condition in terms of the weight function.

In particular, simplifying for $\delta$, we get that:

$$
\left[v_{i}(q+\pi)-v_{i}(q)\right]-\left[v_{i}(p+\pi)-v_{i}(p)\right] \geq 0 \quad \text { for any } p, \pi, i
$$

which, for $\pi$ small enough, becomes:

$$
v_{i}^{\prime}(q)-v_{i}^{\prime}(p) \geq 0 \quad \text { for any } p, i
$$

Having in mind that $p=q+\varepsilon$, this could be written as:

$$
v_{i}^{\prime}(q)-v_{i}^{\prime}(q+\varepsilon) \geq 0 \quad \text { for any } p, i
$$

that for small $\varepsilon$ is equivalent to:

$$
v_{i}^{\prime \prime}(q) \leq 0 \quad \text { for any } p, i
$$

Therefore, in terms of the weight function, the principle of within-group upside positional transfer sensitivity is equivalent to impose negative second derivatives, namely: $v_{i}^{\prime \prime}(p) \leq 0$ for any $i=1, \ldots, n$, for any $p \in[0,1]$.

As we said before, such condition has been introduced by Aaberge, Havnes and Mogstad (2013). However, its interpretation in terms of a combination of a progressive transfer at 
higher ranks and a regressive transfer at lower ranks has not been made explicit so far. We will exploit it to develop some of the properties listed below, that are specific for the bidimensional setting we are analyzing.

It is worth noticing that each one of the two properties we are going to introduce has a twofold interpretation. Specifically, depending on whether we consider a EDFCPT or a EUFCPT - thus assuming downside versus upside inequality aversion with respect to income - each property is shown to have a different interpretation in terms of the transfer sensitivity adopted with respect to needs. Indeed, when we move from an intra-group to an inter-groups evaluation, each one of the principles encompassed by Properties 5 and 6 may be adapted in order to fit in our needs-based framework.

Property 7.

$$
v_{i}^{\prime \prime}(p) \geq v_{i+1}^{\prime \prime}(p) \text { for any } i=1, \ldots, n-1, \text { for any } p \in[0,1]
$$

This property is coherent with two alternative formulations of the principle of positional transfer sensitivity applied over different groups.

In the former case, we endorse downside inequality aversion not only with respect to income, by letting Property 5 hold, but also with respect to needs. This means that we give priority to a progressive transfer at lower ranks in the income distribution rather than at higher ranks, thereby imposing that a EDFCPT does not lead to a welfare loss, and require that the welfare effect of the same EDFCPT applied over different groups, should not be lower the higher is the needs level of the group. To be more precise, we consider two similar EDFCPT that differ only in the group within which they are applied, since one of them takes place within group $i$, whereas the other takes place within a less needy group, $i+1$, and impose that the former is more welfare improving than the latter, which means that:

$\left[v_{i}(p+\pi)-v_{i}(p)\right]-\left[v_{i}(q+\pi)-v_{i}(q)\right] \geq\left[v_{i+1}(p+\pi)-v_{i+1}(p)\right]-\left[v_{i+1}(q+\pi)-v_{i+1}(q)\right] \quad$ for any $p, \pi, \delta, i$

For small $\pi$, this is equivalent to

$$
v_{i}^{\prime}(q+\varepsilon)-v_{i}^{\prime}(q) \geq v_{i+1}^{\prime}(q+\varepsilon)-v_{i+1}^{\prime}(q) \quad \text { for any } p, \pi, i
$$

For $\varepsilon$ small enough, we have that: 


$$
v_{i}^{\prime \prime}(q) \geq v_{i+1}^{\prime \prime}(q) \quad \text { for any } p, i
$$

Combining this property with Property 5, we have that:

$$
v_{i}^{\prime \prime}(p) \geq v_{i+1}^{\prime \prime}(p) \geq 0 \text { for any } i=1, \ldots, n-1, \text { for any } p \in[0,1]
$$

In the latter case, we endorse a completely opposite view, encompassing upside inequality aversion both with respect to income and needs. In particular, the interest in the inequalities affecting the upper part of the distribution would require to devote a greater attention not only to individuals at higher ranks of the income distribution, thus endorsing the view contained in Property 6, but also to groups characterized by a lower level of needs. In this case, the welfare effect of the same EUFCPT applied over different groups, should not be lower the lower is the needs level of the group, that is:

$-v_{i}(p+\pi)+v_{i}(p)+v_{i}(q+\pi)-v_{i}(q) \leq-v_{i+1}(p+\pi)+v_{i+1}(p)+v_{i+1}(q+\pi)-v_{i+1}(q) \quad$ for any $p, \pi, i$

For small $\pi$ this is equivalent to:

$$
v_{i}^{\prime}(q)-v_{i}^{\prime}(q+\varepsilon) \leq v_{i+1}^{\prime}(q)-v_{i+1}^{\prime}(q+\varepsilon) \quad \text { for any } p, i
$$

which, for small $\varepsilon$, is equivalent to:

$$
v_{i}^{\prime \prime}(q) \geq v_{i+1}^{\prime \prime}(q) \quad \text { for any } p, i
$$

Properties 6 and 7, taken together, require that:

$$
v_{i+1}^{\prime \prime}(p) \leq v_{i}^{\prime \prime}(p) \leq 0 \text { for any } i=1, \ldots, n-1, \text { for any } p \in[0,1]
$$

\section{Property 8.}

$$
v_{i}^{\prime \prime}(p) \leq v_{i+1}^{\prime \prime}(p) \text { for any } i=1, \ldots, n-1, \text { for any } p \in[0,1]
$$


Also this property has two different interpretations, corresponding to two different attitudes towards inequality aversion. We describe each one of them in turn.

In the first case, though being more concerned about inequalities occurring at lower ranks of the income disribution, we may be more sensitive to inequalities occurring in the upper part of the needs distribution. In this case, we give priority to a progressive transfer at lower ranks in the income distribution rather than at higher ranks, hence imposing, according to Property 5, that a EDFCPT does not lead to a welfare loss, but, at the same time, we want such EDFCPT to be more effective the lower is the needs level of the group within which it takes place. In more formal terms, this amounts to imposing that, between two similar EDFCPT taking place respectively within group $i$ and within group $i+1$, the former is less welfare improving than the latter, that is:

$\left[v_{i}(p+\pi)-v_{i}(p)\right]-\left[v_{i}(q+\pi)-v_{i}(q)\right] \leq\left[v_{i+1}(p+\pi)-v_{i+1}(p)\right]-\left[v_{i+1}(q+\pi)-v_{i+1}(q)\right] \quad$ for any $p, \pi, i$

For small $\pi$ this means that:

$$
v_{i}^{\prime}(q+\varepsilon)-v_{i}^{\prime}(q) \leq v_{i+1}^{\prime}(q+\varepsilon)-v_{i+1}^{\prime}(q) \quad \text { for any } p, i
$$

which, for small $\varepsilon$, is equivalent to:

$$
v_{i}^{\prime \prime}(q) \leq v_{i+1}^{\prime \prime}(q) \quad \text { for any } p, i
$$

Properties 5 and 8, taken together, require that:

$$
v_{i+1}^{\prime \prime}(p) \geq v_{i}^{\prime \prime}(p) \geq 0 \text { for any } i=1, \ldots, n-1, \text { for any } p \in[0,1]
$$

Alternatively, although we may be interested in upside inequality aversion with respect to the income component, thereby assuming the validity of Property 6, we might still endorse downside inequality aversion with respect to the non-income component, thus requiring that a EUFCPT be more welfare enhancing if applied to needier groups. To say it in other words, this perspective reflects the idea that we give priority to a progressive transfer at higher ranks in the income distribution rather than at lower ranks, hence imposing that a EUFCPT does not lead to a welfare loss, but, at the same time, we want such EUFCPT to be more effective the higher is the needs level of the group within which 
it takes place. This implies that a EUFCPT has a greater effect if applied to group $i$ rather than group $i+1$, that is:

$-v_{i}(p+\pi)+v_{i}(p)+v_{i}(q+\pi)-v_{i}(q) \geq-v_{i+1}(p+\pi)+v_{i+1}(p)+v_{i+1}(q+\pi)-v_{i+1}(q) \quad$ for any $p, \pi, i$

For small $\pi$ this means that:

$$
v_{i}^{\prime}(q)-v_{i}^{\prime}(q+\varepsilon) \geq v_{i+1}^{\prime}(q)-v_{i+1}^{\prime}(q+\varepsilon) \quad \text { for any } p, i
$$

which, for small $\varepsilon$, is equivalent to:

$$
v_{i}^{\prime \prime}(q) \leq v_{i+1}^{\prime \prime}(q) \quad \text { for any } p, i
$$

Properties 6 and 8, taken together, require that:

$$
v_{i}^{\prime \prime}(p) \leq v_{i+1}^{\prime \prime}(p) \leq 0 \text { for any } i=1, \ldots, n-1, \text { for any } p \in[0,1]
$$

The following families of social welfare functions can be identified on the basis of the properties introduced above:

$\mathbf{W}_{1}$ is the class of SWFs satisfying Properties 1 and 2;

$\mathbf{W}_{2}$ is the class of SWFs satisfying Properties 1, 2, 3, 4;

$\mathbf{W}_{3}$ is the class of SWFs satisfying Properties 1, 2, 3, 4, 5, 7 (downside-downside);

$\mathbf{W}_{4}$ is the class of SWFs satisfying Properties $1,2,3,4,5,8$ and for which $v_{i}^{\prime}(1)=0$ $\forall i$ (downside-upside);

$\mathbf{W}_{3^{*}}$ is the class of SWFs satisfying Properties 1, 2, 3, 4, 6, 8 (upside-downside);

$\mathbf{W}_{4^{*}}$ is the class of SWFs satisfying Properties $1,2,3,4,6,7$ and for which $v_{i}^{\prime}(0)=0$ $\forall i$ (upside-upside).

\section{Dominance conditions}

In this section we present the sequential inverse stochastic dominance conditions for the classes of social welfare functions previously defined. The new results of this paper, defining different versions of the third order sequential inverse stochastic dominace, emerge along with the existing sequential dominance tests proposed by Zoli (2000). 
Proposition 1 (Zoli 2000, Proposition 1) Given two distributions $F$ and $G \in \digamma, W(F) \geq$ $W(G) \forall W \in \boldsymbol{W}_{1}$ if and only if

$$
\sum_{i=1}^{k} \Phi_{i}(p) \geq 0 \forall k=1, \ldots, n, \forall p \in[0,1]
$$

where $\Phi_{i}(p)=q_{i}^{F} F_{i}^{-1}(p)-q_{i}^{G} G_{i}^{-1}(p)$

That is, the necessary and sufficient condition for first order welfare dominance for social welfare functions belonging to the class $\mathbf{W}_{1}$, is a sequential inverse stochastic dominance condition of first order. This requires to carry out a comparison between the two distributions $F$ and $G$ of an average of incomes of every subgroup, weighted according to the relative populaton share $q_{i}$, and sequentially aggregated starting from the neediest group, then adding the following, and so on, until all the subgroups have been aggregated. Notice that the comparison has to be conducted at every percentile $p$. Hence the dominance of $F$ on $G$ must hold at every $p$ and every stage $k$.

Proposition 2 (Zoli 2000, Proposition 2) Given two distributions $F$ and $G \in \digamma, W(F) \geq$ $W(G) \forall W \in \boldsymbol{W}_{2}$ if and only if

$$
\sum_{i=1}^{k} \Psi_{i}(p) \geq 0 \forall k=1, \ldots, n, \forall p \in[0,1]
$$

where $\Psi_{i}(p)=\int_{0}^{p} \Phi_{i}(q) d q$

That is, the necessary and sufficient condition for second order welfare dominance for social welfare functions belonging to the class $\mathbf{W}_{2}$, is a sequential inverse stochastic dominance condition of second order. To interpret this condition, we define the Generalized Lorenz curve for F following the formulation by Gastwirth (1971):

$$
G L_{F}(p)=\int_{0}^{p} F^{-1}(t) d t
$$

Thus, we may rewrite $\Psi_{i}(p)$ explicitly in terms of inverse distributions to have that: 
$\Psi_{i}(p)=\int_{0}^{p} q_{i}^{F} F_{i}^{-1}(t) d t-\int_{0}^{p} q_{i}^{G} G_{i}^{-1}(t) d t=q_{i}^{F} \int_{0}^{p} F_{i}^{-1}(t) d t-q_{i}^{G} \int_{0}^{p} G_{i}^{-1}(t) d t=q_{i}^{F} G L_{F_{i}}(p)-q_{i}^{G} G L_{G_{i}}(p)$

where $G L_{F_{i}}(p)$ and $G L_{G_{i}}(p)$ corresponds to the Generalized Lorenz curves associated to distributions $F$ and $G$. Hence, the dominance of $F$ on $G$ for all SWF in $\mathbf{W}_{2}$, requiring that:

$$
\sum_{i=1}^{k} q_{i}^{F} G L_{F_{i}}(p) \geq \sum_{i=1}^{k} q_{i}^{G} G L_{G_{i}}(p) \forall k=1, \ldots, n, \forall p \in[0,1]
$$

means that we have to compare, at every percentile $p$ and every stage $k$ of the sequential procedure, the Generalized Lorenz curves of every subgroup, weighted according to the relative population share $q_{i}$.

Proposition 3 (Zoli 2000, Proposition 3) Given two distributions $F$ and $G \in \digamma, W(F) \geq$ $W(G) \forall W \in \boldsymbol{W}_{3}$ if and only if

$$
\sum_{i=1}^{k} \Psi_{i}(1) \geq 0 \forall k=1, \ldots, n \text { and } \sum_{i=1}^{k} \Gamma_{i}(p) \geq 0 \forall k=1, \ldots, n, \forall p \in[0,1]
$$

where $\Gamma_{i}(p)=\int_{0}^{p} \Psi_{i}(q) d q$

That is, the necessary and sufficient condition for third order welfare dominance for social welfare functions belonging to the class $\mathbf{W}_{3}$, is a sequential upward inverse stochastic dominance condition of third order. In order to check whether such dominance criterion holds, we have to perform two different tests.

As far as the first test is concerned, recall that, generally speaking, $G L(1)=\mu$. Therefore $\sum_{i=1}^{k} \Psi_{i}(1) \geq 0 \forall k=1, \ldots, n$ means that we have to compare, between the two distributions $F$ and $G$, the weighted averages of mean incomes of every subgroup, sequentially aggregated starting from the neediest group, then adding the second, and so on, that is $\sum_{i=1}^{k} q_{i}^{F} \mu\left(F_{i}\right) \geq \sum_{i=1}^{k} q_{i}^{G} \mu\left(G_{i}\right)$. 
As regards the second test, checking whether $\sum_{i=1}^{k} \Gamma_{i}(p) \geq 0 \forall k=1, \ldots, n, \forall p \in[0,1]$, amounts to comparing, at every percentile $p$ and every stage $k$ of the sequential procedure, the integrated Generalized Lorenz curves of every subgroup, integrated from below, weighted according to the relative population share $q_{i}$. This could be expressed also as:

$$
\sum_{i=1}^{k} q_{i}^{F} \int_{0}^{p} G L_{F}(p) \geq \sum_{i=1}^{k} q_{i}^{G} \int_{0}^{p} G L_{G}(p) \forall k=1, \ldots, n, \forall p \in[0,1]
$$

Notice that, endorsing dowside inequality aversion both with respect to income and needs, leads us to:

i) integrate Generalized Lorenz curves starting from the poorest income percentile, that is $p=0$;

ii) aggregate needs groups in the sequential procedure starting from the neediest one, that is $i=1^{2}$.

Proposition 4 Given two distributions $F$ and $G \in \digamma, W(F) \geq W(G) \forall W \in \boldsymbol{W}_{3^{*}}$ if and only if

$$
\sum_{i=1}^{k} \Psi_{i}(1) \geq 0 \forall k=1, \ldots, n \text { and } \sum_{i=1}^{k} \Omega_{i}(p) \geq 0 \forall k=1, \ldots, n, \forall p \in[0,1]
$$

where $\Omega_{i}(p)=\int_{p}^{1} \Psi_{i}(q) d q$

That is, the necessary and sufficient condition for third order welfare dominance for social welfare functions belonging to the class $\mathbf{W}_{3^{*}}$, is a sequential downward inverse stochastic dominance condition of third order.

Proof. We want to find a necessary and sufficient condition for

$$
\Delta W=\sum_{i=1}^{n} \int_{0}^{1} v_{i}(p) \Phi_{i}(p) d p \geq 0 \quad \forall W \in \quad \mathbf{W}_{3^{*}}
$$

where $\Phi_{i}(p)=q_{i}^{F} F_{i}^{-1}(p)-q_{i}^{G} G_{i}^{-1}(p)$

\footnotetext{
${ }^{2}$ For the proofs of Propositions 1, 2 and 3, see Zoli (2000).
} 
In order to prove sufficiency, we will make use of Lemma 1, known as Abel's Lemma, that we now state:

Lemma 1 If $v_{1} \geq \ldots \geq v_{i} \geq \ldots \geq v_{n} \geq 0$, a sufficient condition for $\sum_{i=1}^{n} v_{i} w_{i} \geq 0$ is $\sum_{i=1}^{k} w_{i} \geq 0 \forall k=1, \ldots, n$. If $v_{1} \leq \ldots \leq v_{i} \leq \ldots \leq v_{n} \leq 0$, the same condition is sufficient for $\sum_{i=1}^{n} v_{i} w_{i} \leq 0$

We now turn to the sufficiency proof. First of all, reverse the order of integration and summation in (2) to have

$$
\Delta W=\int_{0}^{1} \sum_{i=1}^{n} v_{i}(p) \Phi_{i}(p) d p \geq 0
$$

Using integration by parts, we can rewrite (3) as

$$
\Delta W=\sum_{i=1}^{n}\left[v_{i}(p) \int_{0}^{p} \Phi_{i}(q) d q\right]_{0}^{1}-\sum_{i=1}^{n} \int_{0}^{1} v_{i}^{\prime}(p) \int_{0}^{p} \Phi_{i}(q) d q d p
$$

Simplifying and substituting for $\Psi_{i}(p)=\int_{0}^{p} \Phi_{i}(q) d q$, we get that

$$
\Delta W=\sum_{i=1}^{n} v_{i}(1) \Psi_{i}(1)-\sum_{i=1}^{n} \int_{0}^{1} v_{i}^{\prime}(p) \Psi_{i}(p) d p
$$

Reversing the order of integration and summation in the second part of (5) and integrating by parts again in the second part of (3.5), we can rewrite $\Delta W$ as follows

$$
\Delta W=\sum_{i=1}^{n} v_{i}(1) \Psi_{i}(1)-\left\{-\sum_{i=1}^{n}\left[v_{i}^{\prime}(p) \int_{p}^{1} \Psi_{i}(q) d q\right]_{0}^{1}+\sum_{i=1}^{n} \int_{0}^{1} v_{i}^{\prime \prime}(p) \int_{p}^{1} \Psi_{i}(q) d q d p\right\}
$$


Simplifying and substituting for $\Omega_{i}(p)=\int_{p}^{1} \Psi_{i}(q) d q$, we get the following expression

$$
\Delta W=\sum_{i=1}^{n} v_{i}(1) \Psi_{i}(1)-\sum_{i=1}^{n} v_{i}^{\prime}(0) \Omega_{i}(0)-\sum_{i=1}^{n} \int_{0}^{1} v_{i}^{\prime \prime}(p) \Omega_{i}(p) d p
$$

By Properties 1 and 2, we can apply Abel's Lemma to get that a sufficient condition for $\sum_{i=1}^{n} v_{i}(1) \Psi_{i}(1) \geq 0$ is that $\sum_{i=1}^{k} \Psi_{i}(1) \geq 0 \forall k=1, \ldots, n$. Moreover, by properties 6 and 8, we can apply Abel's Lemma to get that a sufficient condition for $-\sum_{i=1}^{n} \int_{0}^{1} v_{i}^{\prime \prime}(p) \Omega_{i}(p) d p$ $\geq 0$ is that $\sum_{i=1}^{k} \Omega_{i}(p) \geq 0 \forall k=1, \ldots, n, \forall p \in[0,1]$. Now, since $\mathbf{W}_{3^{*}}$ satisfies also properties 3 and $4, \sum_{i=1}^{k} \Omega_{i}(p) \geq 0 \forall k=1, \ldots, n, \forall p \in[0,1]$ is sufficient also for $-\sum_{i=1}^{n} v_{i}^{\prime}(0) \Omega_{i}(0) \geq 0$.

To show necessity, we follow the same line of reasoning adopted by Palmisano and Peragine (2015). First of all, we resort to reduction to absurd arguments and make use of Lemma 1 in Chambaz and Maurin (1998), Lemma 1 in Atkinson and Bourguignon (1987) and Abel's decomposition, according to which $\sum_{i=1}^{n} v_{i} w_{i}=v_{n} \sum_{i=1}^{n} w_{i}+\sum_{i=1}^{n-1}\left(v_{i}-v_{i+1}\right) \sum_{k=1}^{i} w_{k}$.

Let $\varepsilon_{n}(p)=-v_{n}^{\prime \prime}(p) \forall p$ and $\omega_{i}(p)=-\left(v_{i}^{\prime \prime}(p)-v_{i+1}^{\prime \prime}(p)\right) \forall i, \forall p$. We can apply Abel's decomposition to equation (7) and rewrite it as follows

$$
\Delta W=\sum_{i=1}^{n} v_{i}(1) \Psi_{i}(1)-\sum_{i=1}^{n} v_{i}^{\prime}(0) \Omega_{i}(0)+\int_{0}^{1} \varepsilon_{n}(p) \sum_{i=1}^{n} \Omega_{i}(p) d p+\int_{0}^{1} \sum_{i=1}^{n-1} \omega_{i}(p) \sum_{k=1}^{i} \Omega_{i}(p) d p
$$

Suppose for a contradiction that $\Delta W \geq 0$, but $\exists h \in\{1, \ldots, n-1\}$ and $\exists h=$ $n$ and an interval $I \equiv[a, b] \subseteq[0,1]$ such that $\sum_{k=1}^{h} \Omega_{k}(p)<0 \forall p \in I$. Given that $\left\{\omega_{i}(p) \geq 0\right\}_{i \in\{1, \ldots, n-1\}}$, by Lemma 1 in Chambaz and Maurin (1998) we have that $\sum_{i=1}^{n-1} \omega_{i}(p)\left(\sum_{k=1}^{i} \Omega_{k}(p) d p\right)<$ $0 \forall p \in I$ and given that $\varepsilon_{n}(p) \geq 0$, by Lemma 1 in Atkinson and Bourguignon (1987) 
we have that $\int_{0}^{1} \varepsilon_{n}(p) \sum_{k=1}^{n} \Omega_{k}(p) d p<0$. Denoting $R(p)=\sum_{i=1}^{n-1} \omega_{i}(p)\left(\sum_{k=1}^{i} \Omega_{k}(p) d p\right)$ and $Q(p)=\varepsilon_{n}(p) \sum_{k=1}^{n} \Omega_{k}(p) d p$, we have

$$
\Delta W=\sum_{i=1}^{n} v_{i}(1) \Psi_{i}(1)-\sum_{i=1}^{n} v_{i}^{\prime}(0) \Omega_{i}(0)+\int_{0}^{1} Q(p) d p+\int_{0}^{1} R(p) d p
$$

If we choose $R(p)$ such that $R(p) \rightarrow 0$ for some $p \in[0,1] \backslash I$, we have that:

$$
\Delta W=\sum_{i=1}^{n} v_{i}(1) \Psi_{i}(1)-\sum_{i=1}^{n} v_{i}^{\prime}(0) \Omega_{i}(0)+\int_{0}^{1} Q(p) d p+\int_{a}^{b} R(p) d p
$$

Furthermore, suppose for a contradiction that there exists some $j$ for which $\sum_{i=1}^{j} \Psi_{i}(1)<$ 0 .

Given that $\int_{a}^{b} R(p) d p<0$ and $\int_{0}^{1} Q(p) d p<0$, we can always choose a combination of $v_{i}(1)$ and $\Psi_{i}(1)$, or a combination of $v_{i}^{\prime}(0)$ and $\Omega_{i}(0)$,for which $\Delta W<0$, that is a contradiction.

Here again we interpret separately the two parts of Proposition 4. The first condition of Proposition 4 is identical to the one that we have introduced before, namely $\sum_{i=1}^{k} q_{i}^{F} \mu\left(F_{i}\right) \geq$ $\sum_{i=1}^{k} q_{i}^{G} \mu\left(G_{i}\right)$

The second condition is dual to the second part of Proposition 3 insofar as it endorses the opposite view about the integration procedure. In fact, checking whether $\sum_{i=1}^{k} \Omega_{i}(p) \geq$ $0 \forall k=1, \ldots, n, \forall p \in[0,1]$, amounts to comparing, at every percentile $p$ and every stage $k$ of the sequential procedure, the integrated Generalized Lorenz curves of every subgroup, integrated from above, weighted according to the relative population share $q_{i}$. This is equivalent to: 


$$
\sum_{i=1}^{k} q_{i}^{F} \int_{p}^{1} G L_{F}(p) \geq \sum_{i=1}^{k} q_{i}^{G} \int_{p}^{1} G L_{G}(p) \forall k=1, \ldots, n, \forall p \in[0,1]
$$

Therefore, first we have to compare the integrated GL, integrated from above, and weighted according to $q_{i}$, for the neediest group, $i=1$, at every $p$. Then, we have to add the second group, $i=2$, and so on, up to the least needy group, i.e. $i=n$, and perform the same check at every step.

In this case, we are assuming upside inequality aversion with respect to income and downside inequality aversion with respect to needs. This attitude is suitable for a framework in which, in order to check for third order inverse sequential stochastic dominance, we have to:

i) integrate Generalized Lorenz curves starting from the richest income percentile, i.e. $p=1$;

ii) aggregate needs groups in the sequential procedure starting from the neediest one, i.e. $i=1$.

Proposition 5 Given two distributions $F$ and $G \in \digamma$, a sufficient condition for $W(F) \geq$ $W(G) \forall W \in \boldsymbol{W}_{4}$ is

$$
\sum_{i=1}^{k} \Psi_{i}(1) \geq 0 \forall k=1, \ldots, n \text { and } \sum_{i=n}^{k} \Gamma_{i}(p) \geq 0 \forall k=n, n-1, \ldots, 1, \forall p \in[0,1]
$$

where $\Gamma_{i}(p)=\int_{0}^{p} \Psi_{i}(q) d q$

Proof. We want to find a sufficient condition for

$$
\Delta W=\sum_{i=1}^{n} \int_{0}^{1} v_{i}(p) \Phi_{i}(p) d p \geq 0 \quad \forall W \in \mathbf{W}_{4}
$$

where $\Phi_{i}(p)=q_{i}^{F} F_{i}^{-1}(p)-q_{i}^{G} G_{i}^{-1}(p)$

We propose Lemma 2, representing an alternative formulation of Abel's Lemma:

Lemma 2 If $v_{n} \geq \ldots \geq v_{i} \geq \ldots \geq v_{1} \geq 0$, a sufficient condition for $\sum_{i=1}^{n} v_{i} w_{i} \geq 0$ is 
$\sum_{i=n}^{k} w_{i} \geq 0 \forall k=n, n-1, \ldots, 1$. If $v_{n} \leq \ldots \leq v_{i} \leq \ldots \leq v_{1} \leq 0$, the same condition is sufficient for $\sum_{i=1}^{n} v_{i} w_{i} \leq 0$.

We now turn to the sufficiency proof. Reversing the order of integration and summation in the second part of (3.11) and integrating by parts again in the second part of (11), we can rewrite $\Delta W$ as follows

$$
\Delta W=\sum_{i=1}^{n} v_{i}(1) \Psi_{i}(1)-\sum_{i=1}^{n}\left[v_{i}^{\prime}(p) \int_{0}^{p} \Psi_{i}(q) d q\right]_{0}^{1}+\sum_{i=1}^{n} \int_{0}^{1} v_{i}^{\prime \prime}(p) \int_{0}^{p} \Psi_{i}(q) d q d p
$$

Simplifying and substituting for $\Gamma_{i}(p)=\int_{0}^{p} \Psi_{i}(q) d q$, we get the following expression:

$$
\Delta W=\sum_{i=1}^{n} v_{i}(1) \Psi_{i}(1)-\sum_{i=1}^{n} v_{i}^{\prime}(1) \Gamma_{i}(1)+\sum_{i=1}^{n} \int_{0}^{1} v_{i}^{\prime \prime}(p) \Gamma_{i}(p) d p
$$

Since we assumed that all $W \in \mathbf{W}_{4}$ satisfies $v_{i}^{\prime}(1)=0 \forall i$, expression (13) becomes:

$$
\Delta W=\sum_{i=1}^{n} v_{i}(1) \Psi_{i}(1)+\sum_{i=1}^{n} \int_{0}^{1} v_{i}^{\prime \prime}(p) \Gamma_{i}(p) d p
$$

By properties 1 and 2, we can apply Abel's Lemma to get that a sufficient condition for $\sum_{i=1}^{n} v_{i}(1) \Psi_{i}(1) \geq 0$ is that $\sum_{i=1}^{k} \Psi_{i}(1) \geq 0 \forall k=1, \ldots, n$. Moreover, by properties 5 and 8 , according to which $v_{i+1}^{\prime \prime}(p) \geq v_{i}^{\prime \prime}(p) \geq 0$ for any $i=1, \ldots, n-1$, for any $p \in[0,1]$, we can apply Lemma 2 to get that a sufficient condition for $\sum_{i=1}^{n} \int_{0}^{1} v_{i}^{\prime \prime}(p) \Gamma_{i}(p) d p \geq 0$ is that $\sum_{i=n}^{k} \Gamma_{i}(p) \geq 0 \forall k=n, n-1, \ldots, 1, \forall p \in[0,1]$ 
Also this proposition is composed of two tests: the former is identical to the first tests in Propositions 3 and 4 ; the latter, asking to check whether $\sum_{i=n}^{k} \Gamma_{i}(p) \geq 0 \forall k=$ $n, n-1, \ldots, 1, \forall p \in[0,1]$, is similar to the second test in Proposition 3, except for the fact that it requires to carry out the sequential procedure starting from the last group rather than from the first. Hence, first we have to compare the integrated GL, integrated from below, and weighted according to $q_{i}$, for the least needy group, $i=n$, at every $p$. Then, we have to add group $i=n-1$, and so on, up to the neediest group, $i=1$, and perform the same check at every step. This could be expressed also as:

$$
\sum_{i=n}^{k} q_{i}^{F} \int_{0}^{p} G L_{F}(p) \geq \sum_{i=n}^{k} q_{i}^{G} \int_{0}^{p} G L_{G}(p) \forall k=n, n-1, \ldots, 1, \forall p \in[0,1]
$$

If we endorse downside inequality aversion with respect to income and upside inequality aversion with respect to needs, then we come up with a third order procedure in which we:

i) integrate Generalized Lorenz curves starting from the poorest income percentile, that is $p=0$;

ii) aggregate needs groups in the sequential procedure starting from the least needy one, that is $i=n$.

Proposition 6 Given two distributions $F$ and $G \in \digamma$, a sufficient condition for $W(F) \geq$ $W(G) \forall W \in \boldsymbol{W}_{4^{*}}$ is

$$
\sum_{i=1}^{k} \Psi_{i}(1) \geq 0 \forall k=1, \ldots, n \text { and } \sum_{i=n}^{k} \Omega_{i}(p) \geq 0 \forall k=n, n-1, \ldots, 1, \forall p \in[0,1]
$$

where $\Omega_{i}(p)=\int_{p}^{1} \Psi_{i}(q) d q$

Proof. We want to find a sufficient condition for

$$
\Delta W=\sum_{i=1}^{n} \int_{0}^{1} v_{i}(p) \Phi_{i}(p) d p \geq 0 \quad \forall W \in \mathbf{W}_{4^{*}}
$$

where $\Phi_{i}(p)=q_{i}^{F} F_{i}^{-1}(p)-q_{i}^{G} G_{i}^{-1}(p)$ 
Let us consider again exression (7), i.e.

$\Delta W=\sum_{i=1}^{n} v_{i}(1) \Psi_{i}(1)-\sum_{i=1}^{n} v_{i}^{\prime}(0) \Omega_{i}(0)-\sum_{i=1}^{n} \int_{0}^{1} v_{i}^{\prime \prime}(p) \Omega_{i}(p) d p$

By assumption, $v_{i}^{\prime}(0)=0 \forall i$ holds for all $W \in \mathbf{W}_{4^{*}}$, therefore we have:

$$
\Delta W=\sum_{i=1}^{n} v_{i}(1) \Psi_{i}(1)-\sum_{i=1}^{n} \int_{0}^{1} v_{i}^{\prime \prime}(p) \Omega_{i}(p) d p
$$

By Properties 1 and 2, we can apply Abel's Lemma to get that a sufficient condition for $\sum_{i=1}^{n} v_{i}(1) \Psi_{i}(1) \geq 0$ is that $\sum_{i=1}^{k} \Psi_{i}(1) \geq 0 \forall k=1, \ldots, n$. Moreover, since $v_{i+1}^{\prime \prime}(p) \leq v_{i}^{\prime \prime}(p) \leq 0$ for any $i=1, \ldots, n-1$, for any $p \in[0,1]$, we can apply Lemma 2 to get that a sufficient condition for $-\sum_{i=1}^{n} \int_{0}^{1} v_{i}^{\prime \prime}(p) \Omega_{i}(p) d p \geq 0$ is that $\sum_{i=n}^{k} \Omega_{i}(p) \geq 0 \forall k=n, n-1, \ldots, 1, \forall p \in[0,1]$.

Two tests characterize Proposition 6: the former is common to Propositions 3, 4 and 5; the latter, asking to check whether $\sum_{i=n}^{k} \Omega_{i}(p) \geq 0 \forall k=n, n-1, \ldots, 1, \forall p \in[0,1]$, is similar to the second test in Proposition 4, except for the fact that it requires to carry out the sequential procedure starting from the last group rather than from the first. Hence, first we have to compare the integrated GL, integrated from above, and weighted according to $q_{i}$, for the least needy group, $i=n$, at every $p$. Then, we have to add group $i=n-1$, and so on, up to the neediest group, $i=1$, and perform the same check at every step. This could be expressed also as:

$$
\sum_{i=n}^{k} q_{i}^{F} \int_{p}^{1} G L_{F}(p) \geq \sum_{i=n}^{k} q_{i}^{G} \int_{p}^{1} G L_{G}(p) \forall k=n, n-1, \ldots, 1, \forall p \in[0,1]
$$

This is the last possible attitude towards inequality aversion, supporting upside inequality aversion both with respect to income and needs. In this case, to check whether third order inverse sequential stochastic dominance holds, we have to:

i) integrate Generalized Lorenz curves starting from the richest income percentile, i.e. $p=1$; 
ii) aggregate needs groups in the sequential procedure starting from the least needy one, i.e. $i=n$.

\section{Concluding remarks}

Several contributions in the economic literature show the need to modify the standard framework to rank income distributions, in order to take into account non-income aspects of well-being. At the same time, an increasing interest has been shown for inequalities affecting the upper part of the income distribution. In this context, the choice between upside and downside inequality aversion specifies whether, in the evaluation of social welfare, one should give priority to equalizing transfers between poorer vis-a-vis richer individuals.

This work represents an attempt to bring together these issues, by introducing upside inequality aversion considerations within a bidimensional evaluation of social welfare. In particular, we adopt a rank-dependent and needs-based social welfare function and develop third order dominance conditions to rank bidimensional distributions when a concern for upside inequality aversion is embedded.

To this end, we propose some properties that our SWF should satisfy. These properties have the common feature of presenting different formulations of a principle, introduced by Aaberge (2009) and called upside positional transfer sensitivity, that has been discussed only in terms of the unidimensional distribution of income. We enrich the literature by adapting such principle to a bidimensional context, in order to provide an alternative framework for those who might be more concerned about differences in the upper part of the distribution rather than in the lower part of it. In particular, we propose different combinations of upside and dowside inequality aversion referred alternatively to the income and the non-income component, to come up with a complete framework allowing for various possible normative choices. We intoduce three different third order stochastic dominance conditions, holding for classes of social welfare functions that are built upon such choices.

\section{References}

[1] Aaberge, R., 2000. "Characterizations of Lorenz curves and income distributions", Social Choice and Welfare 17, 639-653. 
[2] Aaberge, R., 2009. "Ranking intersecting Lorenz curves", Social Choice and Welfare $33(2), 235-259$.

[3] Aaberge, R., Havnes, T. and M. Mogstad, 2013. "A theory for ranking distribution functions", Discussion Papers No. 763, Statistics Norway, Research Department.

[4] Aaberge, R. and E. Peluso, 2012. "A counting approach for measuring multidimensional deprivation", IZA Discussion Papers 6589, Institute for the Study of Labor (IZA).

[5] Atkinson, A.B., 1970. "On the measurement of inequality", Journal of Economic Theory $2,244-263$.

[6] Atkinson, A.B. and F. Bourguignon, 1987. "Income distribution and differences in needs", in G. R. Feiwel (ed.), Arrow and the foundations of the theory of economic policy. London: Macmillan, chapter 12 .

[7] Atkinson, A.B. and T. Piketty, 2007 (eds.). "Top incomes over the twentieth century: a contrast between continental European and English-speaking Countries", Oxford University Press.

[8] Atkinson, A.B. and T. Piketty, 2010 (eds.). "Top Incomes: A Global Perspective", Oxford University Press.

[9] Chambaz, C. and E. Maurin, 1998. "Atkinson and Bourguignon's dominance criteria: extended and applied to the measurement of poverty in France", Review of Income and Wealth 4, 497-513.

[10] Dardanoni, V. and P.J. Lambert, 1988. "Welfare rankings of income distributions: a role for the variance and some insights for tax reform", Social Choice and Welfare 5, $1-17$.

[11] Davies, J. and M. Hoy, 1994. "The normative significance of using third-degree stochastic dominance in comparing income distributions", Journal of Economic Theory 64(2), 520-530.

[12] Davies, J. and M. Hoy, 1995. "Making inequality comparisons when Lorenz curves intersect", American Economic Review, 85(4), 980-86. 
[13] Duclos, J.Y. and D. Échevin, 2009. "Health and income: A robust comparison of Canada and the US", Working Paper 09-09, CIRPÉE.

[14] Gastwirth, J.L., 1971. "A general definition of the Lorenz curve", Econometrica 39, 1037-1039.

[15] Jenkins, S. and P.J. Lambert, 1993. "Ranking income distributions when needs differ", Review of Income and Wealth 39, 337-356.

[16] Kolm, S.C., 1976a. "Unequal inequalities. I", Journal of economic theory 12, 416-442.

[17] Kolm, S.C., 1976b. "Unequal inequalities. II", Journal of economic theory 13, 82-111.

[18] Lambert, P.J. and X. Ramos, 2002. "Welfare comparisons: sequential procedures for heterogeneous populations", Economica 69(276), 549-562.

[19] Mehran, F., 1976. "Linear measures of income inequality", Econometrica 44, 805-809.

[20] Muliere, P. and M. Scarsini, 1989. "A note on stochastic dominance and inequality measures", Journal of Economic Theory 49, 314-323.

[21] Palmisano, F. and V. Peragine, 2015. "The distributional incidence of growth: a social welfare approach", Review of Income and Wealth 61(3), 440-464.

[22] Shorrocks, A.F., 1983. "Ranking income distributions", Economica 50, 3-17.

[23] Shorrocks, A.F. and J.E. Foster, 1987. "Transfer sensitive inequality measures", Review of Economic Studies 14, 485-497.

[24] Weymark, J.A., 1981. "Generalized Gini inequality indices", Mathematical Social Sciences 1, 409-430.

[25] Yaari, M.E., 1987. "The dual theory of choice under risk", Econometrica 55, 99-115.

[26] Yaari, M.E., 1988. "A controversial proposal concerning inequality measurement", Journal of Economic Theory 44, 381-397.

[27] Zoli, C., 1999. "Intersecting generalized Lorenz curves and the Gini index", Social Choice and Welfare 16, 183-196. 
[28] Zoli, C., 2000. "Inverse sequential stochastic dominance: rank-dependent welfare, deprivation and poverty measurement", Discussion Paper in Economics No. 00/11, University of Nottingham. 